\title{
YAP/TFEB pathway promotes autophagic cell death and hypertrophic cardiomyopathy in lysosomal storage diseases
}

\author{
Inna Rabinovich-Nikitin ${ }^{1}$ and Lorrie A. Kirshenbaum ${ }^{1,2}$ \\ 'The Institute of Cardiovascular Sciences, St. Boniface Hospital Albrechtsen Research Centre, Department of Physiology and Pathophysiology and ${ }^{2}$ Department of Pharmacology and Therapeutics Rady \\ College of Medicine, Max Rady Faculty of Health Sciences, University of Manitoba, Winnipeg, Manitoba, Canada.
}

\begin{abstract}
Lysosomal storage disorders (LSD) are a group of inherited metabolic diseases characterized by lysosomal enzyme deficiency. The cardiac phenotype includes cardiomyopathy with eventual heart failure. Lysosomemediated degradation processes, such as autophagy, maintain cellular homeostasis by discarding cellular debris and damaged organelles. Under stress, the transcription factor EB (TFEB) moves into the nucleus to activate transcription of lysosome biogenesis and autophagic proteins. In this issue of the JCl, Ikeda et al. report on their exploration of the signaling pathway involved with regulating lysosomal proteins specifically in the heart. The researchers generated a mouse model for LSD that was restricted to cardiac tissue. Unexpectedly, modulation of TFEB alone was insufficient to fully rescue the underlying clearance defect in lysosomal-associated disorders. The authors identified the Yes-associated protein (YAP)/TFEB signaling pathway as a key regulator of autophagosomes. These findings suggest that undigested autophagosomes accumulate and result in the cell death and cardiac dysfunction observed with LSD.
\end{abstract}

\section{Regulating quality-control mechanisms}

Lysosomes are membrane-bound organelles that contain acid-rich hydrolases that digest damaged biomolecules, oxidized proteins, and lipids and therefore play a pivotal role in maintaining cellular hemostasis. Lysosome-mediated degradation processes, such as autophagy, are essential quality-control mechanisms that discard cellular debris and damaged organelles, such as mitochondria, from the cell. For example, autophagosomal cargo destined for clearance is rapidly hydrolyzed upon fusion of the matured autophagosome with lysosomes (1).

The transcription factor EB (TFEB) is a master regulator of lysosome biogenesis and autophagic proteins. Under basal conditions, TFEB is localized in the cytosol; however, under stress conditions, TFEB translocates to the nucleus where it activates transcription of genes encoding lysosomal hydrolases, proteins involved in lysosome biogenesis, and several autophagic factors (2). Notably, defects in lysosomal-regulated TFEB activity have detrimental effects on many cellular processes. Specifically, lysosomal dysfunction caused by proteotoxic stress and impaired protein clearance is highly correlated with cardiac dysfunction following ischemia-reperfusion $(\mathrm{I} / \mathrm{R})$ and end-stage heart failure. During I/R, the liberation of cellular debris and accumulation of oxidized proteins from tissue necrosis is a major underlying cause of contractile failure. However, over-

Related Article: https://doi.org/10.1172/JCl143173

Conflict of interest: The authors have declared that no conflict of interest exists.

expression of TFEB in postischemic cardiomyocytes promoted cell survival and decreased myocardial infarct size, presumably by restoring autophagy-lysosome clearance of cellular debris (3). These findings demonstrate the importance of TFEB as a crucial regulator of the lysosomemediated quality-control mechanism for protecting cardiac myocytes against proteotoxic stress and accumulation of cellular debris during I/R.

Consistent with this notion, lysosomal storage disorders (LSD) are a group of inherited metabolic diseases characterized by a deficiency in lysosomal enzymes and accumulation of aggregated proteins and damaged organelles. The cardiac phenotype of LSD includes hypertrophic and dilated cardiomyopathy, coronary artery disorders, and valvular defects that eventually manifest as heart failure (4). A recent study showed that overexpression of TFEB in myotubes and muscle fibers in Pompe disease, a subtype of LSD, increased the formation and clearance of autophagolysosomes by enhancing lysosomal and cell-membrane fusion (5). Accordingly, the functional restoration of TFEB abrogated the inherent lysosomal clearance in these myotubes by increasing lysosome biogenesis and lysosomal-autophagosomal fusion. These findings support the concept that reactivating lysosomal activity through gain of function of TFEB activity could potentially rescue the autophagy clearance defect in individuals with LSD.

\section{Contradicting the TFEB rescue paradigm}

Remarkably, however, in this issue of the JCI, Ikeda et al. (6) contradict the paradigm that TFEB rescues autophagy defects and suggest that modulation of TFEB alone is insufficient to fully rescue the underlying clearance defect in lysosomal-associated 
A

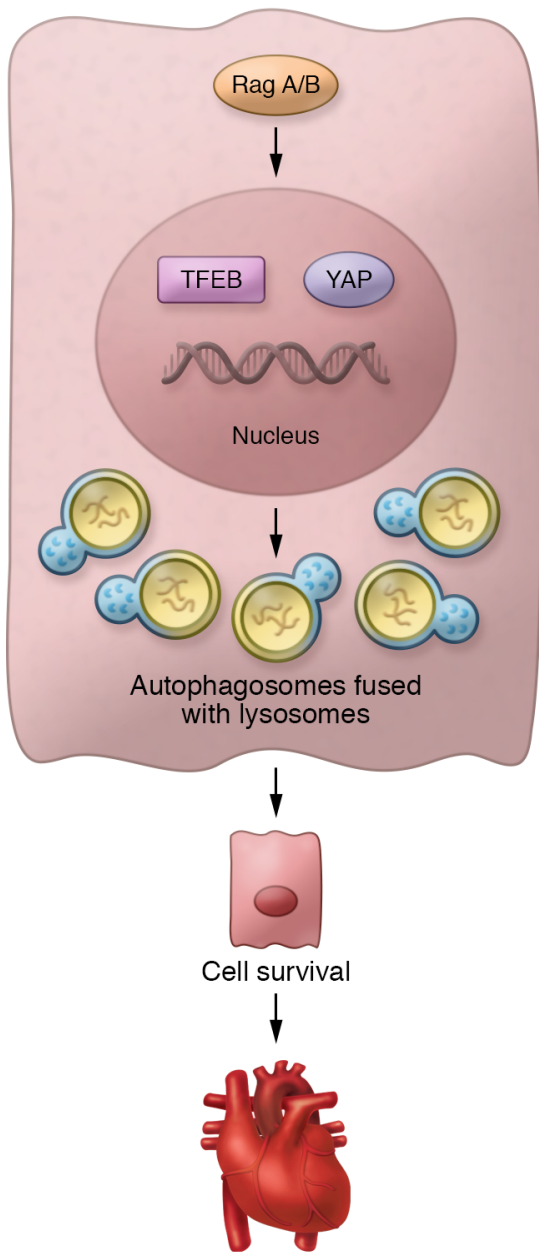

Normal heart
B

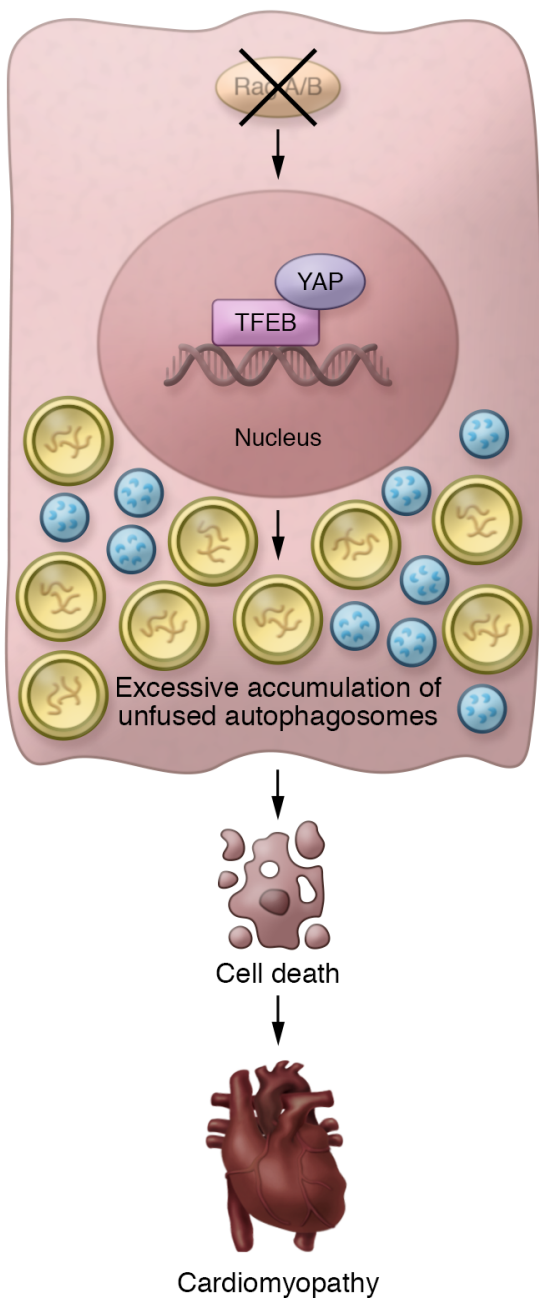

Figure 1. Model for autophagy and lysosomal degradation in the regulation of LSD. During knockdown of RagA/B, YAP interacts with TFEB, causing accumulation of autophagosomes, decreased cardiac function, and enhanced cell death.

disorders. In fact, according to the study by Ikeda et al. (6), defects in lysosomal function caused by genetic mutations, such as those found in LSD, require not only efforts to enhance autophagosome formation by TFEB, but also the simultaneous upregulation of lysosomal function to ensure that the newly formed autophagosomes are effectively cleared from the cell.

In order to validate this hypothesis, Ikeda et al. (6) used a cardiac-specific RagA/B cKO mouse model of LSD, in which lysosomal dysfunction induces cardiomyopathy. Notably, RagA and RagB are GTPases found on the lysosome outer membrane that are critical for the docking and fusion of autophagosome membrane with lysosomal membranes, a step necessary for hydrolysis of the autophagosomal cargo (7).
Notably, the authors found that in this mouse model, the Yes-associated protein (YAP), a transcription cofactor and a major effector of the Hippo-signaling pathway, was highly expressed in the hearts (6). The Hippo/YAP signaling pathway is an evolutionary conserved pathway involved in regulating organ size, growth, and apoptosis (8). Consistently, YAP not only has been shown to influence aberrant growth and cell proliferation linked to tumorigenesis, but has also been implicated in tissue repair, fibrosis, and heart hypertrophy $(9,10)$. In the current study, constitutive YAP activation in the RagA/B cKO mice coincided with excessive accumulation of autophagosomes, dedifferentiation of the cardiac phenotype, and contractile dysfunction. The accumulation of autopha- gosomes in response to YAP activation, occurred in the presence of bafilomycin, an autophagy flux inhibitor that inhibits lysosome acidification and autophagosome-lysosome fusion.

The accumulation of autophagosomes in response to YAP activation in the RagA/B cKO mice resulted in a corresponding increase in cardiac cell death from the activation of apoptotic and necrotic death programs. This finding prompted the authors to investigate whether normalization of YAP activity would rescue lysosomal dysfunction and cell death in Rag A/B-deficient cardiac myocytes. Using reciprocal approaches to either up or downregulate YAP expression, the authors surprisingly found that neither gain nor loss of YAP function had any effect on lysosomal acidification, indicating YAP does not directly influence lysosome function. These results were consistent with the fact that YAP increased LC3II levels, indicative of autophagy activation, without affecting lysosome function in RagA/BcKO cardiomyocytes (6).

\section{Recapitulating the detrimental effects of YAP}

Based on the YAP-expression findings, the authors realized that YAP alone could not be the sole driver of lysosomal function and next investigated the mechanism by which YAP affects autophagosome formation (6). The rationale for their next experiment was based on a previous work that showed loss of function of RagA and RagB in cardiomyocytes resulted in development of hypertrophic cardiomyopathy, a finding consistent with the heart hypertrophy in LSD with constitutive TFEB activation (11). Following the TFEB line, Ikeda et al. (6) tested to determine whether TFEB is responsible for mediating YAP-regulated cardiomyocyte death and autophagosomal dysfunction in the RagA/B cKO hearts. Indeed, in vitro analysis of cardiomyocytes transduced with or without Ad-sh-RagA/B revealed that while both YAP and TFEB translocated into the nucleus, YAP unexpectedly formed protein complexes with TFEB following RagA/B knockdown. These finding suggest that interaction of YAP with TFEB activates TEFB-dependent transcription, resulting in the accumulation of autophagosome de novo in the absence of effective lysosomal clearance, leading to cardiac myocyte death. This conclusion was further supported by 
the finding that TAT-Beclin 1, which activated autophagy, exacerbated cardiomyopathy in the RagA/B cKO mice - recapitulating the detrimental effects of YAP (6).

There is growing evidence that accumulation of autophagosomes without concurrent autophagic clearance is toxic to cardiac myocytes and activates multiple forms of programmed cell death (1214). To validate this notion, the authors supressed autophagy by knocking down Atg7 in cardiac myocytes in conjunction with sh-RagA/B. Interestingly, Atg7 knockdown not only decreased the number of autophagosomes, but also supressed cardiac myocyte cell death. Furthermore, when RagA/B cKO mice were crossed with Atg7 heterozygous cKO mice, cardiac hypertrophy and LV dysfunction normalized. In contrast, however, cardiac dysfunction in RagA/B cKO mice following autophagy activation with TAT-Beclin 1 exacerbated cardiac injury. The combination of TAT-Beclin 1 along bafilomycin increased cardiac myocyte size, while enhancing accumulation of autophagosomes, supporting the view that defective lysosomal clearance resulting in autophagosome accumulation promotes maladaptive cardiac growth and hypertrophy (6).

\section{Clinical implications}

The study by Ikeda et al. (6) strongly suggests that autophagy and lysosomal degradation play a pivotal role in the regulation of LSD. The authors identified the YAP/TFEB signaling pathway as a key regulator of autophagosomes such that lysosome clearance with the accumulation of undigested autophagosomes underlies cell death and cardiac dysfunction in LSD (Figure 1). Prior to this study, stimulation of lysosomal biogenesis with TFEB or other means was viewed as the most reasonable treatment for cardiac disorders associated with lysosomal dysfunction, such as LSD. However, Ikeda et al. (6) contravene this dogma and suggests instead that increasing autophagosome biogenesis in the absence of simultaneous activation of lysosomal clearance pathways exacerbates cardiac dysfunction from the accumulation of undigested autophagosomes. It is noteworthy that many of the in vivo models developed for LSD over the past 25 years (15) fail to fully recapitulate human LSD disease. Therefore, although the current study suggests a different approach for treating LSD is warranted, it remains to be determined why previous studies in which TFEB-mediated autophagy was enhanced were shown to be effective in LSD.

Additional studies are needed to address the interplay between the Hippo/ YAP pathway and TFEB in other cell processes, such as cell growth, proliferation, and fibrosis. Further studies should also determine whether targeted therapies that selectively modulate YAP/TFEB activity will be viable therapeutic options for mitigating other disease entities involving Hippo/YAP beyond LSD. For example, while autophagy activation following I/R can reportedly be protective or detrimental, the accumulation of autophagosomes from impaired autophagosome and lysosome degradation has been associated with cardiac dysfunction and heart failure. Notably, cardiac deficiency from deletion of the mitochondrial dynamic protein mitofusion 2 (MFN2) was shown to impair clearance of damaged mitochondria and resulted in the accumulation of undigested autophagosomes from impaired autophagosome-lysosomal fusion $(16,17)$. These findings highlight the importance of MFN2 as a critical regulator of autophagosome-lysosome clearance. Whether MFN2 or other cellular proteins such as Rabs or SNAREs that are involved in tethering of autophagosomes with lysosomes are influenced by YAP signaling in LSD remains to be determined.

Nevertheless, the conclusion drawn from the present study highlights the complexity of cellular quality control mechanisms involving autophagy and lysosomal storage diseases. Hence, interventions designed to enhance cellular clearance pathways will likely need to target more than one pathway to ensure effective removal of cellular debris without impairing cell viability or organ dysfunction. Since autophagy plays an important role in the pathogenesis of LSD, further studies are needed to resolve the interplay between autophagy regulation and cardiac dysfunction in LSD.

\section{Acknowledgments}

This work was supported by a foundation grant to LAK from the Canadian Institute for Health Research (CIHR) and St. Boniface Hospital Research Foundation. LAK holds a Canada Research Chair in Molecular Cardiology; IRN holds a postdoctoral fellowship from the CIHR.

Address correspondence to: Lorrie A. Kirshenbaum, Institute of Cardiovascular Sciences, St. Boniface Hospital Albrechtsen Research Centre Rm. 3016, 351 Taché Avenue, Winnipeg, Manitoba, Canada, R2H 2A6. Phone: 204.235.3661; Email: lkirshenbaum@sbrc.ca.

1. Parzych KR, Klionsky DJ. An overview of autophagy: morphology, mechanism, and regulation. Antioxid Redox Signal. 2014;20(3):460-473.

2. Di Malta C, et al. Transcriptional regulation of autophagy: Mechanisms and diseases. Front Cell Dev Biol. 2019;7:114

3. Pan B, et al. TFEB activation protects against cardiac proteotoxicity via increasing autophagic flux. JMol Cell Cardiol. 2017;113:51-62.

4. Nair V, et al. Lysosomal storage disorders affecting the heart: a review. Cardiovasc Pathol. 2019;39:12-24

5. Spampanato C, et al. Transcription factor EB (TFEB) is a new therapeutic target for Pompe disease. EMBO Mol Med. 2013;5(5):691-706.

6. Ikeda $S$, et al. YAP plays a crucial role in the development of cardiomyopathy in lysosomal storage diseases. JClin Invest. 2021;131(5):e143173.

7. Sancak Y, et al. Ragulator-Rag complex targets mTORC1 to the lysosomal surface and is necessary for its activation by amino acids. Cell. 2010;141(2):290-303.

8. Wackerhage H, et al. The Hippo signal transduction network in skeletal and cardiac muscle. Sci Signal. 2014;7(337):re4.

9. Wang J, et al. The Hippo pathway in the heart: pivotal roles in development, disease, and regeneration. Nat Rev Cardiol. 2018;15(11):672-684.

10. Landry NM, Dixon IMC. Fibroblast mechanosensing, SKI and Hippo signaling and the cardiac fibroblast phenotype: Looking beyond TGF- $\beta$. Cell Signal. 2020;76:109802.

11. Kim YC, et al. Rag GTPases are cardioprotective by regulating lysosomal function. Nat Commun. 2014;5:4241.

12. Kessel DH, et al. ATG7 deficiency suppresses apoptosis and cell death induced by lysosomal photodamage. Autophagy. 2012;8(9):1333-1341.

13. Bialik S, et al. Autophagy-dependent cell death where, how and why a cell eats itself to death. J Cell Sci. 2018;131(18):jcs215152.

14. Biala AK, Kirshenbaum LA. The interplay between cell death signaling pathways in the heart. Trends Cardiovasc Med. 2014;24(8):325-331.

15. Pastores GM, et al. Animal models for lysosomal storage disorders. Biochemistry (Mosc). 2013;78(7):721-725.

16. Song M, et al. Abrogating mitochondrial dynamics in mouse hearts accelerates mitochondrial senescence. Cell Metab. 2017;26(6):872-883.

17. Zhao T, et al. Central role of mitofusin 2 in autophagosome-lysosome fusion in cardiomyocytes. J Biol Chem. 2012;287(28):23615-23625. 